\title{
Editor's Note: Applied Intelligence and COVID-19 Research
}

Published online: 2 May 2020

(C) Springer Science+Business Media, LLC, part of Springer Nature 2020
The COVID-19 pandemic has impacted the entire world, touching so many areas of individual lives. It has affected the population's health, the capacity of healthcare systems, the way people work, attend school and socialize.

Applied Intelligence has been providing the scientific community with valuable resources for testing and implementing various artificial intelligence, machine learning and data mining methods resulting in best practices in research and theory for high accuracy and robust systems that would fulfill our intelligent demanding society. However, the recent invasion of COVID-19 imposed on us to revise these theories and practices testing it for better accuracy and robustness in terms of Infectious Disease Control and Prediction. The Journal has dedicated itself to a special issue: Artificial Intelligence Applications for COVID-19, detection, Control, Prediction, and Diagnosis (for short as SI-COVID), inviting contributions from various engineering, scientific, and social settings that exploit data analytics, machine learning, and data mining, and other Artificial Intelligence techniques for early diagnosis, and data analytics on human behavior, culture and other types of data distribution for decision making on infectious disease for its control and prediction.

Applied Intelligence welcomes submissions reporting on research results and intermediate outcomes on topics related to COVID applied medical systems, early prediction and diagnosis of COVID-19 from human gathering, studies on the use of face mask wearing prevention data, studies on multi sensing for automatic symptoms analysis and prediction, and relative emerging solution for the COVID-19 outbreak spreading behavior though feature selection, information retrieval, social networking, deep learning and other emerging technologies.

This special issue will comprise an online topical collection to include papers where the pandemic is directly or indirectly related to the research. The connection between the pandemic and submitted papers must be clear and explicit. Submissions will be reviewed using the standard journal guidelines for Applied Intelligence. For more information, please visit: https://www.springer.com/10489. 